JOURNAL DE PHYSIQUE

Coltoque C7, supplément au no 12, Tome 43, décembre 1982

page $\mathrm{C} 7-227$

\title{
DETERMINATION BY POLARIZED NEUTRON DIFFRACTION OF THE SPIN DENSITY DISTRIBUTION IN A NON-CENTROSYMMETRICAL CRYSTAL OF DPPH: ${ }_{6} \mathrm{H}_{6}$
}

\author{
J.X. Boucherle*, B. Gillon ${ }^{* *}$, J. Maruani** and J. Schweizer * \\ * DRF/DN, CENG, B.P. 85X, 38041 Grenoble Cedex, France \\ + ILL, B.P. 156X, 38042 Grenoble Cedex, France \\ * CMOA, 23 me du Maroc, 75019 Paris, France
}

Résumë. - La densité de spin du radica1 libre DPPH. (diphenylepicrylehydrazyle) a été étudiēe dans la forme cristallisée $\mathrm{DPPH}: \mathrm{C}_{6} \mathrm{H}_{6}$, avec les moments paramagnêtiques orientés, à basse température, par un champ magnétique extérieur. Comme 1a structure cristalline est non centrosymétrique, il n'est pas possible d'obtenir directement les facteurs de structure magnétiques à partir des rapports de "flipping" mesurés par diffraction de neutrons polarisés. Pour cette raison, la densité de spin a été représentée par un dêveloppement multipolaire sur chaque atome. Les paramètres de ce développement sont déterminês directement par une comparaison avec les rapports de "flipping" mesurës.

La plus grande partie de la densité de spin est partagée entre les deux atomes d'azote du groupe hydrazyl $\left(N_{\alpha}\right.$ et $\left.N_{\beta}\right)$. La partie restante est délocalisée sur les 2 cycles phenyl et sur le cycle picryl. Ces résultats sont comparês aux mesures de résonance magnêtique.

Abstract. - The spin density of the free radical DPPH (diphenylpicrylhydrazy1) has been studied in the crystallized form $\mathrm{DPPH} \mathrm{C}_{6} \mathrm{H}_{6}$. with the paramagnetic moments orientated, at low temperature, by an external magnetic field. As the crystal structure is not centro-symmetrical, it is not possible to obtain directly the magnetic structure factors from the experimental flipping ratios measured by polarized neutron diffraction. For this reason we have represented the spin density by a multipole expansion on the different atoms, and determined directly the parameters of this expansion by comparing with the observed flipping ratios.

A large amount of the spin density is shared between the 2 nitrogen atoms $\left(\mathrm{N}_{\alpha}\right.$ and $\left.\mathrm{N}_{\beta}\right)$ of the hydrazyl group. The remaining part is delocalized on the 2 phenyl and the picryl rings. These results are compared to magnetic resonance data.

1. Introduction. - The stable aromatic free radical $\alpha, \alpha$-diphenyl $\beta$-picryl hydrazyl (DPPH) was among the first paramagnetic species investigated by electron spin resonance (ESR) [1], and its remarkable chemical stability and narrowness of its resonance line have made it a widespread standard for measuring unpaired spin concentrations and effective gyromagnetic ratios [2]. DPPH crysta11izes in severa1 different forms, with [3a] or without [3b] the inclusion of solvent molecules. Complete $\mathrm{X}$-ray determination of the crystal structure at room temperature was first performed on the best grown crystal form, the DPPH : $\mathrm{C}_{6} \mathrm{H}_{6}$ complex [4], and later extended to acetone-containing DPPH crystals [5], revealing in both cases a rather twisted conformation (Fig. 1, table II).

Fig. 1 - Overa11 view of DPPH structure as deduced from neutron diffraction data at 10K (paragraph 3)

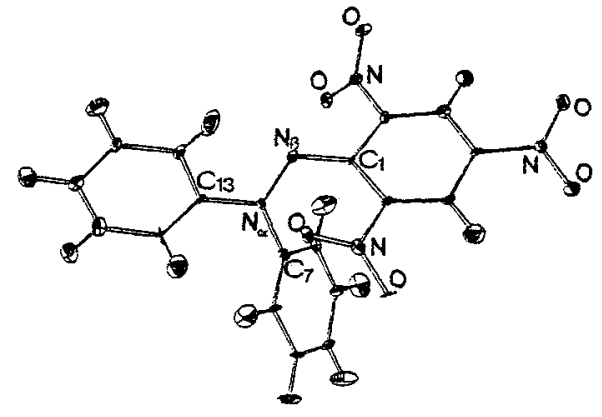


The DPPH molecule is one of the most widely investigated molecules using magnetic resonance techniques [6]. ESR measurements on dilute solutions have mainly yielded the hyperfine couplings of the central nitrogen atoms in a large variety of physical conditions. Both the contact and dipolar parts of these couplings have been shown to depend significantly on the solvent composition, rigidity and structure, and the spin densities to be almost as large on the nitrogen linked to the two phenyls as on the nitrogen linked to the picryl [7]. Nuclear magnetic resonance (NMR) of concentrated samples has essentially provided the magnitudes and signs of the hyperfine couplings of the ring protons [8] and of the picryl nitrogens [9], revealing an extensive spin delocalization and polarization on the whole molecule. Contact hyperfine couplings have been determined most accurately by electron-electron double resonance (ELDOR) for the hydrazyl nitrogens [10] and by electron-nuclear double resonance (ENDOR) for the ring protons [11]. The hydrazyl nitrogens appear to share about $70 \%$ of the total spin density, the remaining part being delocalized on the 2 phenyl and the picryl rings, with alternating positive and negative signs on the ring carbons, in agreement with quantum mechanical calculations [12].

One remarkable feature of the magnetic resonance results for the hydrazyl nitrogens is that their hyperfine coupling tensors appear consistently quasiaxial and nearly parallel [7], whereas, in the $f$ ew cases where the exact molecular geometry has been determined from $X$-ray diffraction data [4,5], the corresponding trigonal planes are at an ang $1 \mathrm{e}$ close to $30^{\circ}$.

Table I shows some sets of values obtained for the hyperfine couplings of DPPH under various conditions. The first column summarizes the most accurate values measured for the contact couplings using appropriate techniques. The second column recalls the values measured in dilute glassy samples by ESR [7b] and in pure crystalline powders by NMR [8a]. The third column provides the corresponding values for DPPH : $\mathrm{C}_{6} \mathrm{H}_{6}$ in dilute $[7 a, c]$ or pure [8c] crystals. The signs and assignments are confirmed by the theoretical results given in the fourth column; here, we have used the UHF spin populations $\rho^{\pi}$ calculated for an extended structure [12b] together with the semiempirical relations :

$$
a_{\mathrm{H}}=\mathrm{Q}_{\mathrm{CH}} \rho_{\mathrm{C}}^{\pi} \quad \mathrm{a}_{\mathrm{N}}=\mathrm{Q}_{\mathrm{NN}} \rho_{\mathrm{N}}^{\pi}
$$

with the spin polarization constants :

$$
Q_{\mathrm{CH}}=-23 \mathrm{G} \text { [13a], } \quad \mathrm{Q}_{\mathrm{N \alpha}}=36 \mathrm{G} \text { and } \mathrm{Q}_{\mathrm{N} \beta}=25 \mathrm{G}[12 \mathrm{~b}], \mathrm{Q}_{\mathrm{NO}_{2}}=48 \mathrm{G}[13 \mathrm{~b}]
$$

As has been shown for nitroxide free radicals [14], the direct determination of the spin density distribution by neutron diffraction techniques can reveal new and important results, complementary to those obtained with magnetic resonance techniques. Contrary to nitroxide radicals, where the spin density is concentrated on the $\mathrm{N}-\mathrm{O}$ group, DPPH offers a case of large delocalization, which makes it a stimulating cha1lenge for neutron diffraction. Unfortunately, all the crystal forms of DPPH are noncentrosymmetrical and this has prevented any study with polarized neutrons to be done so far.

Recently a new technique for data reduction has been proposed [15], which makes use of a multipolar expansion for the spin density. This method makes it possible to study non-centrosymmetrical crystals by polarized neutron diffraction. We have undertaken such an experiment on a DPPH single crystal, the results of which are reported in this paper.

In the following Section we show how multipolar expansions make it possible to measure spin densities in non-centrosymmetrical crystals. Section 3 describes the determination of the crystal structure of the DPPH: $\mathrm{C}_{6} \mathrm{H}_{6}$ complex using unpolarized neutron diffraction. In Section 4 we describe the polarized neutron experiments and the derivation of spin density maps. In the last Section we discuss the results of neutron diffraction in relation to those of magnetic resonance. 


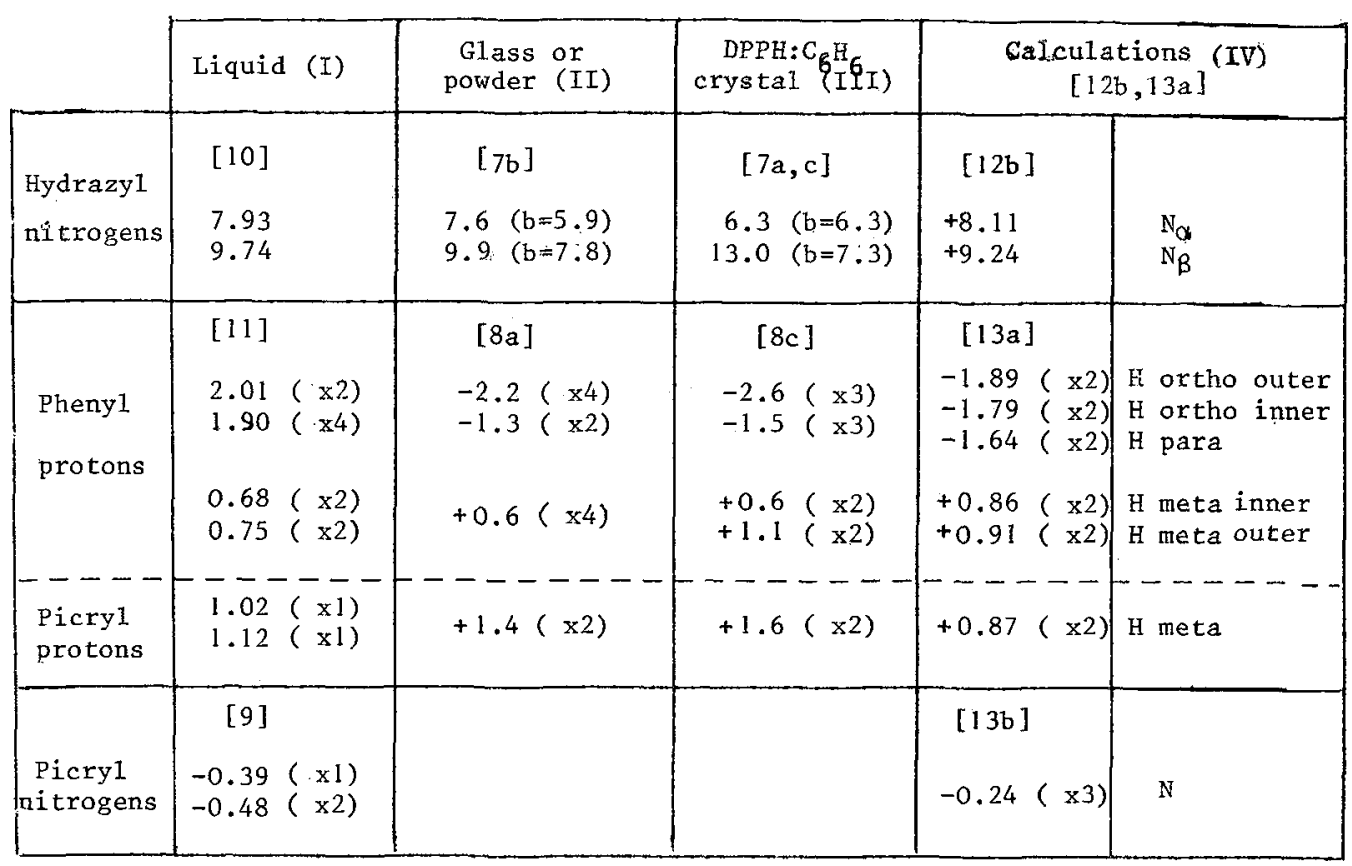

Table I : Contact hyperfine coupling a (in Gauss) of the nitrogen and hydrogen nuclei in DPPH (the numbers of nuclei are given in parenthesis).

I - ELDOR [10] and ENDOR [11] do not provide the coupling signs whereas NMR [9] does. II, IIr - For $N_{\alpha}, \beta$ one obtains also the dipolar coupling b (in Gauss) by ESR [7a,b] and for the H's, the intermediate value of the anisotropic tensor by NMR [8a,c]. The measured values for the hyperfine couplings of the hydrazyl nitrogens range from $a=6.3, b=5.0$ to $a=8.7, b=7.0$ for $N_{\alpha}$ and $a=9.4, b=5.0$ to $a=13.0, b=8.0$ for $N_{\beta}[6]$. IV - UHF calculation for planar DPPH.

\section{Measurement of spin density distributions in non-centrosymmetrical crystals - Multipolat expansion}

The neutron intensity of a Bragg reflection for a magnetic material contains both the nuclear structure factor $F_{N}$ and the magnetic structure factor $F_{M}$. Accurate determinations of magnetic stricture factors are usually performed with polarized neutrons and the flipping ratio technique. One measures, for each reflection, the ratio $R$ obtained for two neutron spin states up and down

$$
\mathrm{R}=\frac{\mathrm{I}^{+}}{\mathrm{I}}-=\frac{\left|\mathrm{F}_{\mathrm{N}}+\mathrm{F}_{\mathrm{M}}\right|^{2}}{\left|\mathrm{~F}_{\mathrm{N}}-\mathrm{F}_{\mathrm{M}}\right|^{2}}
$$

If the nuclear crystal structure of the sample under investigation has been determined, then the nuclear structure factor $F_{N}$ is known and hence $F_{M}$ may be deduced from measurement of $R$.

In the case of centro symmetrical crystals, for which $F_{N}$ and $F_{M}$ are real, $F_{M}$ is obtained by solving equation (2). In the case of non-centrosymmetrical crystals, both $F_{N}$ and $F_{M}$ are complex quantities

$$
\mathrm{F}_{\mathrm{N}}=\mathrm{F}_{\mathrm{N}}^{\prime}+\mathrm{i} \mathrm{F}_{\mathrm{N}}^{\prime \prime} \quad \mathrm{F}_{\mathrm{M}}=\mathrm{F}_{\mathrm{M}}^{\prime}+\mathrm{i} \mathrm{F}_{\mathrm{M}}^{\prime \prime}
$$

and the expression for the flipping ratio becomes : 


$$
R=\frac{\left(F_{N}^{\prime}+F_{M}^{\prime}\right)^{2}+\left(F_{N}^{\prime \prime}+F_{M}^{\prime \prime}\right)^{2}}{\left(F_{N}^{\prime}-F_{M}^{\prime}\right)^{2}+\left(F_{N}^{\prime \prime}-F_{M}^{\prime \prime}\right)^{2}}
$$

The measurements of $R$ alone cannot provide a knowledge of the two unknown quantities $F_{M}^{\prime}$ and $F_{M}^{\prime \prime}$. To overcome this difficulty we propose to apply a model which provides an analytical description of the spin density. We have chosen an expansion of the density around the nuclei at rest. Such a model, first used to represent charge densities, has already proved to be very well adapted in the case of spin densities in centrosymmetric crystals. The expansion consists of a superposition of aspherical atomic densities, each described by a series expansion in real spherical harmonic functions :

$$
s(\vec{r})=\sum_{\text {atoms }} \sum_{\ell=0}^{\infty} R_{\ell}(r) \sum_{m=-\ell}^{\ell} \mathrm{P}_{\ell m} \mathrm{Y}_{\ell m}(\vec{r})
$$

The $P_{\ell m}$ are population coefficients. The radial functions $R_{\ell}(r)$ are of Slater type :

$$
R_{\ell}(r)=\frac{\zeta^{n+3}}{(n+r) !} r^{n} e^{-\zeta r}
$$

The magnetic structure factors corresponding to eqs. (1) become

$$
\begin{gathered}
F_{M}(\overrightarrow{\mathrm{H}})=\sum_{\text {atoms }} e^{2 \pi i \overrightarrow{\mathrm{H}} \cdot \vec{r}}\left[\sum_{\ell=0}^{\infty} \phi_{\ell}(\mathrm{H}) \sum_{\mathrm{m}=-\ell}^{\ell} \mathrm{P}_{\ell \mathrm{m}} \mathrm{Y}_{\ell \mathrm{m}}(\hat{\mathrm{H}})\right] \mathrm{e}^{-\mathrm{w}} \\
\text { with } \phi_{\ell}(\mathrm{H})=4 \pi i^{\ell} \int_{0}^{\infty} R_{\ell}(\mathrm{r}) \mathrm{j}_{\ell}(2 \pi \mathrm{Hr}) \mathrm{r}^{2} \mathrm{dr} \mathbf{r}
\end{gathered}
$$

To establish the spin density map, the set of parameters $\left(\zeta, \mathrm{P}_{\mathrm{lm}}\right)$ which characterize the spin density and fit the experimental data must be determined. To do this, given a set of parameters, $F_{M}^{\prime}$ and $F \dot{M}$, are calculated and a value $R_{c a l}$ for the flipping ratio of each measured reflection is obtained from equations (3). By comparing $R_{c a l}$ with the measured flipping ratios $R_{o b s}$ it is possible to refine the parameters ( $\zeta$, $\left.P_{k m}\right)$ which best fit the data.

\section{The crystal structure of the $\mathrm{DPPH}: \mathrm{C}_{6} \mathrm{H}_{6}$ complex}

DPPH is paramagnetic and its magnetic susceptibility follows a Curie law, with a magnetic moment of $1 \mu_{\mathrm{B}}$ per molecule. For the present investigation, one intends to measure the magneticamplitude $F_{M}\left(F_{M}=F_{M}^{\prime}+i F_{M}^{\prime \prime}\right)$ scattered by the component of the magnetic moment aligned by an external magnetic field, and compare it with the nuclear amplitude $F_{N}\left(F_{N}=F_{N}+i F_{N}^{\prime \prime}\right)$ scattered by all the nuclei of the DPPH molecule. The magnetic moment is small compared to the nuclear signal, and only part of it is aligned by the field. To obtain a degree of alignnent large enough to make the magnetic signal measurable, it is necessary to perform the neutron diffraction experiment at very low temperature, in order to take advantage of the increase of susceptibility with decreasing temperature.

At very low temperatures, however, the static susceptibility reveals strong deviations from the Curie law, due to antiferromagnetic exchange coupling between adjacent molecules : for pure DPPH crystals a maximum occurs around 10K [17a], whereas for the DPPH- ${ }_{6} \mathrm{H}_{6}$ complex (where the free radicals are kept apart by the benzene molecules) the susceptibility reaches a maximum around $1 \mathrm{~K}$ [17b] (see Figure 2). This latter form is therefore better suited for a polarized neutron study made at the helium temperature $\mathrm{T}=4.2 \mathrm{~K}$.

As all other forms of $\mathrm{DPPH}$, the $\mathrm{DPPH}: \mathrm{C}_{6} \mathrm{H}_{6}$ complex is non-centrosymmetrical : its space group is Pc with 2 molecules of both $\mathrm{BPPH}$ and $\mathrm{C}_{6}{ }_{6}{ }_{6}$ in the crystal cell $(\mathrm{Z}=2)$. For the spin density determination, the crystal structure, as determined at room temperature by $\mathrm{X}$-rays [4] is not accurate enough. In particular one has to know the location of the hydrogen nuclei and also the exact values of all the thermal para- 


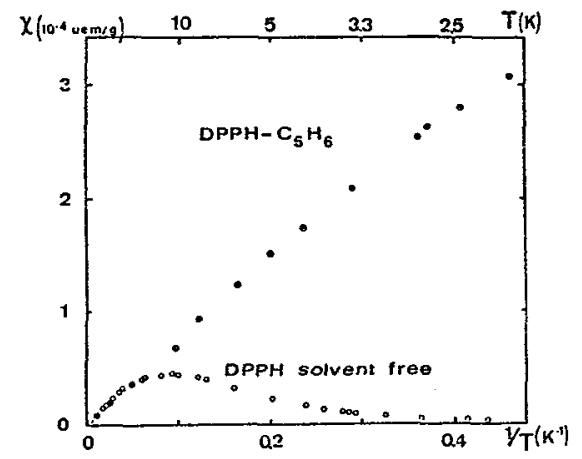

Fig. 2 - Magnetic susceptibility

$X$ as a function of the inverse of the temperature $1 / \mathrm{T}$ for $\mathrm{DPPH}$ solvent free and for the DPPH: $\mathrm{C}_{6} \mathrm{H}_{6}$ complex $[17 \mathrm{a}, \mathrm{b}]$

meters at the temperature for which the spin density is measured. For this reason, we have first undertaken a low temperature unpolarized neutron crystal study, on a DPPH- ${ }_{6}{ }_{6}{ }_{6}$ single crystal.

This experiment was performed on the 4 circle diffractometer D15 of the ILL. The temperature, produced by a two stage refrigerator, was $10 \mathrm{~K}$. The lattice constants at this temperature are reported in Table II. 1270 independent reflections were collected and corrected for absorption. We have refined the positions and anisotropic thermal parameters of all atoms in the cell. The final weighted agreement factor for a11 reflections was $3.6 \%$.

The main features of the crystal structure thus measured are similar to those reported by Williams (Fig. 1, Table II). The main difference between the 2 sets of results is in a systematic contraction of bond lengths at 10K. The deviation from planarity of the environment of $\mathrm{N}_{\alpha}$ is about $3^{\circ}$. The 2 phenyl rings are completely planar, but with different twist angles with respect to the $\mathrm{N}_{\alpha}$ plane.

\begin{tabular}{|c|c|c|c|}
\hline & & Reference [4] & This work \\
\hline 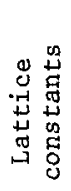 & $\begin{array}{l}a \\
b \\
c \\
B\end{array}$ & $\begin{array}{r}7.764 \AA \\
10.648 \AA \\
14.780 \AA \\
109.05^{\circ}\end{array}$ & $\begin{array}{r}7.542 \AA \\
10.416 \AA \\
14.295 \AA \\
108.80^{\circ}\end{array}$ \\
\hline 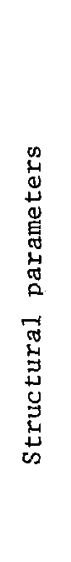 & 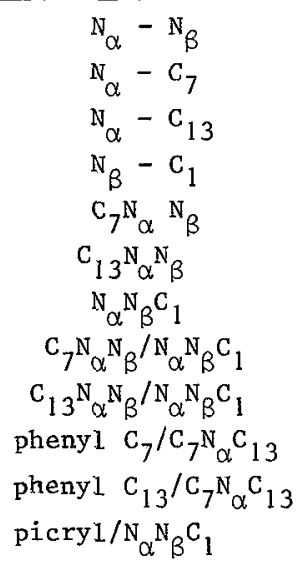 & $\begin{array}{l}1.334 \AA \\
1.422 \AA \\
1.419 \AA \\
1.370 \AA \\
121.8^{\circ} \\
115.1^{\circ} \\
118.5^{\circ} \\
34.4^{\circ} \\
23.2^{\circ} \\
54.3^{\circ} \\
15.6^{\circ} \\
32.9^{\circ}\end{array}$ & $\begin{array}{r}1.318 \AA \\
1.416 \AA \\
1.395 \AA \\
1.337 \AA \\
121.7^{\circ} \\
116.3^{\circ} \\
119.2^{\circ} \\
33.6^{\circ} \\
22.8^{\circ} \\
54.3^{\circ} \\
14.9^{\circ} \\
33.7^{\circ}\end{array}$ \\
\hline
\end{tabular}

Table II : Lattice constants, bond lengths, planar angles and dihedral angles defining the structure of DPPH syncrystallized with $\mathrm{C}_{6} \mathrm{H}_{6}$ as determined by X-rays at room temperature [4] and by neutrons at $10 \mathrm{~K}$. 
Fina11y, as it was reported [4] that a certain amount of benzene may evaporate from the mixed crystals, we have refined the exact stoichiometry of the $\mathrm{C}_{6} \mathrm{H}_{6}$ molecules, and found exactly $1: 1$. As our crystals had been stored at $-20^{\circ} \mathrm{C}$, we concluded this was enough to prevent evaporation.

\section{Polarized neutron measurements and data treatment}

The polarized neutron experiment was performed at a temperature of $4.2 \mathrm{~K}$, with an applied magnetic field of $4.65 \mathrm{~T}$ provided by a cryomagnet on the D3 diffractometer of the ILL Grenoble. The wavelength of the neutron beam was $0.90 \AA$. The sample was a single crystal of $\mathrm{DPPH}: \mathrm{C}_{6}{ }_{\mathrm{H}} 6$, of dimensions $1 \times 2 \times 6 \mathrm{~mm}$, with the long dimension close to the $\vec{a}$ axis of the cel1. The crystal was oriented with this axis vertical, parallel to the magnetic field. The flipping ratios of 425 inequivalent Bragg reflections were measured, lifting when necessary the counter above the horizontal plane to measure in layers other than the zero layer.

The treatment of the flipping ratios was conducted as explained in Section II. The spin density was expanded in atomic multipoles according to relation (4), and the parameters $\zeta$ and $P_{l m}$ were refined. Experimental corrections due to imperfect polarization and flipping efficiency, $\lambda / 2$ contamination and nuclear polarization of hydrogen atoms were.included, modifying slightly relation (3). At the present time no diamagnetic contribution to the magnetization density has been removed. This remains to be done.

In the following, we present partial results on the spin density, obtained with multipoles limited to $l=2$. No constraint was imposed to the central nitrogens, but the spin density on the carbon atoms was constrained to their $2 \mathrm{p}_{\mathrm{z}}$ orbitals, perpendicular to the ring. No spin density was examined on the $\mathrm{NO}_{2}$ groups. The total number of parameters to be refined was 39 . The least square refinement corresponds to $\chi^{2}=2.76$. The spin density map, projected along $\vec{c}$, is represented in Figure 3 .

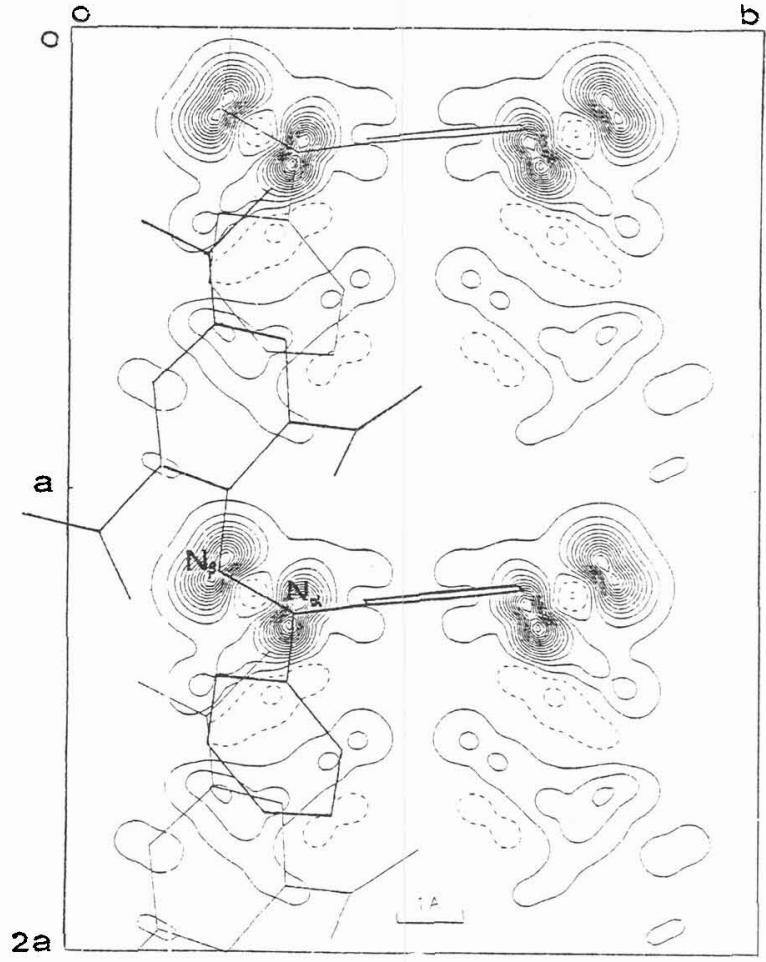

Fig. 3 - Projection of the spin densitydistribution along the erystallographic axis $\vec{c}$. Adjacent contours are separated by $0.03 \mu_{B} / \AA^{2}$.

\section{Discussion}

As seen in the map, the largest amount of spin density is located on the two central nitrogen atoms. However, and contrary to the case of nitroxide radicals [14], 30\% of the spin density is delocalized on the two phenyl and the picryl rings.

The spin distribution over the different parts of the molecule is illustrated by the 3 projection maps given in Figure 4. The spin density of the hydrazyl (Fig. 4a) is projected perpendicularly to the plane containing the $\mathrm{N}_{\alpha}-\mathrm{N}_{\beta}$ bond and bissecting the $\mathrm{C}_{7} \mathrm{~N}_{6} \mathrm{C}_{13}$ angle. It is well separated into two $2 \mathrm{p}$-type atomic orbitals and evenly distributed between them. At the present stage of data refinement the axes of the 2 spin distributions appear nearly parallel, whereas the local structure is twisted by over $28^{\circ}$ (Figure 1, Table ID). This may be linked to the very low value observed by ESR for the angle between the axes of the hyperfine coupling tensors of the two central nitrogens [7]. However, departure of hyperfine coup1ings from the local structure has been attributed in other systems [18] to dipolar interactions of the considered nucleus with the spin densities surrounding neighbouring nuclei. 


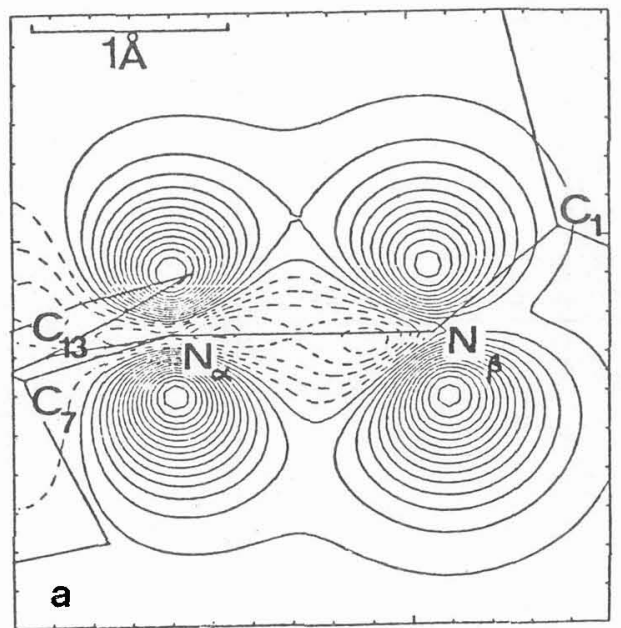

Fig. 4 - Projection of the spin density

a) along a direction parallel to the trigonal $\mathrm{N}_{\alpha}$ plane

b) perpendicularly to the phenyl ring $\left(\mathrm{C}_{13}\right)$

c) perpendicularly to the phenyl ring $\left(\mathrm{C}_{7}\right)$

Adjacent contours are separated by $0.03 \mu_{B} / \AA^{2}$.

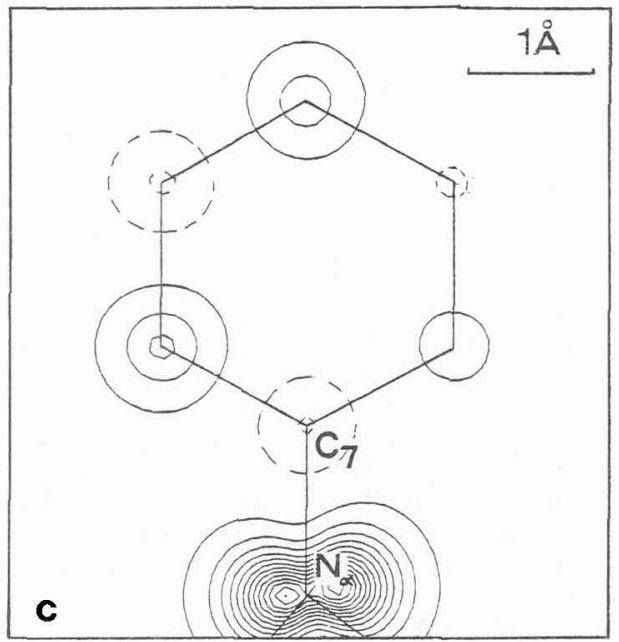

Delocalization of the spin density has been observed on the carbon atoms of the three rings of the molecule, in accordance with the magnetic resonance data. The projection maps onto the 2 phenyl planes (Fig. 4b and 4c) show that the spin density on the carbon atoms is alternatively positive and negative, as predicted by theoretical calculations including spin polarization [12b] and in agreement with NMR results $[8 \mathrm{a}, 8 \mathrm{c}]$. Table III compares the spin populations of the $2 \mathrm{p}^{\pi}$ orbitals for each carbon, as deduced from the hyperfine coupling constants measured on $D P P H: \mathrm{C}_{6} \mathrm{H}_{6}$ crystals [8c] by the use of McConnel1's relation ( 1 ), to the monopole populations $P_{\text {oo }}$ after normalization. All values fit into this scheme and the agreement is quite good. This can be considered as a confirmation of McConnell's constant $\mathrm{Q}_{\mathrm{CH}}=-23 \mathrm{G}$.

Moreover, the inequivalence of the two phenyl rings, already noticed in the NMR of single crystals [8c], is clearly illustrated in Figures $4 \mathrm{~b}$ and $4 \mathrm{c}$. The spin populations located on the carbon atoms of phenyl ring $\left(C_{13}\right)$, which makes a tilt angle of $15^{\circ}$ with regard to the $N_{\alpha}$ trigonal plane, are about twice as large as those relative to phenyl ring $\left(C_{7}\right)$, whose tilt angle is $54^{\circ}$. These delocalized spin populations increase with the overlap between the $2 \mathrm{p}^{\pi}\left(\mathrm{N}_{\alpha}\right)$ and $2 \mathrm{p}^{\pi}(\mathrm{C})$ orbitals [19], that is with $\cos ^{2} \alpha$, where $\alpha$ is the twist angle. Resonance results in DPPH solutions [11], where 
no tilt angle can be defined, do not permit to detect an inequivalence between the two phenyl rings.

Table III: Spin populations $\rho^{\pi}$ of the $2 p^{\pi}$ orbital of the carbon atoms of DPPH :

I - deduced from the experimental couplings in DPPH-C $6{ }_{6}{ }_{6}$ [8c] by use of the

McConne11 relation ( 1 )

II - given by the normalized monopole population of each atom in the multipole representation of the spin density

\begin{tabular}{|c|c|c|c|c|}
\hline \multicolumn{2}{|l|}{ NMR [8c] } & \multicolumn{3}{|c|}{ neutrons (this work) (II) } \\
\hline+0.113 & (3) & $\begin{array}{l}+0.083 \\
+0.083 \\
+0.074\end{array}$ & $\begin{array}{l}\text { ortho } \\
\text { para } \\
\text { ortho }\end{array}$ & phenyl $C_{13}$ \\
\hline+0.065 & (3) & $\begin{array}{l}+0.064 \\
+0.049 \\
+0.020\end{array}$ & $\begin{array}{l}\text { ortho } \\
\text { para } \\
\text { ortho }\end{array}$ & pheny $1 \mathrm{C}_{7}$ \\
\hline-0.026 & $(2)$ & $\begin{array}{l}-0.015 \\
-0.039\end{array}$ & $\begin{array}{l}\text { meta } \\
\text { meta }\end{array}$ & phenyl $\mathrm{C}_{7}$ \\
\hline-0.048 & (2) & $\begin{array}{l}-0.044 \\
-0.049\end{array}$ & $\begin{array}{l}\text { meta } \\
\text { meta }\end{array}$ & pheny $1 C_{13}$ \\
\hline-0.071 & $(2)$ & $\begin{array}{l}+0.034 \\
-0.005\end{array}$ & $\begin{array}{l}\text { meta } \\
\text { meta }\end{array}$ & picryl $c_{1}$ \\
\hline
\end{tabular}

\section{References}

[1] Holden A.N., Kittel C., Merritt F.R., and Yager W.A., Phys. Rev. 77 (1950) 147 ; Townes C.H., and Turkevich J., ibid. 148.

[2] Buchachenko A.L., Stable Radica1s, \$ IV 1a (Consu1tants Bureau, New York) 1965.

[3] (a) Sternberg M., C.R. Acad. Sci. 240 (1955) 990 ; (b) Williams D.E., J. Chem. Soc. (19.65)7535.

[4] Williams D.E., J. Amer. Chem. Soc. 88 (1966) 5665 ; 89 (1967) 4280.

[5] Kiers C.Th., de Boer J.L., O1thof R., and Spek A.L., Acta Cryst. B32 (1976)2297

[6] Maruani J., Magnetic Resonance and Related Techniques, in Becker P., "Electron and Magnetization Densities in Molecules and Crystals", pp. 633-692 (Plenum Press, New York) 1980

[7] (a) Holmberg R.W., Livingston R., and Smith Jr. W.T., J. Chem. Phys. 33 (1960) 541 ; (b) Sane K.V. and Wei1 J.A., Proc. Co11. AMPERE XI (1962) 431 ; (c) Lefebvre R., Maruani J. and Marx R., J. Chem. Phys. 41 (1964) 585.

[8] (a) Anderson M.E., Pake G.E. and Tuttle Jr. T.R., J. Chem. Phys. 33 (1960) 1581. (b) Sagdeev R.Z., Molin Yu. N., Koryakov V.I., Chirkov A.K. and Matevosyan R.0., Org. Magn. Reson. 4 (1972) 365 ; (c) Yoshioka T., Ohya-Nishiguchi H., and Deguchi.Y., BuIl Chem. Soc. Japan 47 (1974) 430.

[9] Dalal N.S., Ripmeester J.A., and Reddoch A.H., J. Magn. Reson. 31 (1978) 471

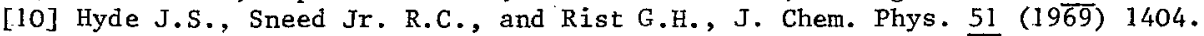

[11] Dalal N.S., Kennedy D.E., and McDowel1 C.A., J. Chem. Phys. 59 (1973) 3403

[12] (a) Wa1ter R.I., J. Amer. Chem. Soc. 88 (1966) 1930 ; (b) Gubanov V.A., Koryakov V.I., Chirkov A.K., and Matevosyan R.0., J. Struct. Chem. 11 (1970) 941 .

[13] (a) McConnel1 H.M. and Chesnut D.B., J. Chem. Phys. 28 (1958) 107 ; (b) Nanda D.N., Subramanian J. and Narasimhan P.T., Theor. Chim. Acta 22 (1971) 369.

[14] Brown P.J., Capiomont A., Gillon B., and Schweizer J., J. Magn. \& Magn. Mat. 14 (1979) 289 .

[15] Boucherle J. X., Gillon B., and Schweizer J., Proc. Int. Symp. Neutron Scattering, Am.Inst.Phys.-New York (1982) pp. 333 .

[16] Hansen N.K. and Coppens P., Acta Cryst. A 34 (1978) 909.

[17] (a) Grobet P., Van Gerven L., and Van den Bosch A., J. Chem. Phys. 68 (1978) 522.

(b) Duffy Jr. W., Strandburg D.L., and Deck J.F., J. Chem. Phys. 68 (1978) 2097.

[18] (a) Heller C. and Cole T., J. Chem. Phys. 37 (1962) 243 ; (b) Edlund 0. , Lund A., Shiotani M., Sohma J., and Thuomas K. $-\AA$., Mo1. Phys. 32 (1976) 49.

[19] McLachlan A.D., Mol.Phys. I (1958) 233. 